\title{
Chest CT score in COVID-19 patients: correlation with disease severity and short-term prognosis
}

\author{
Marco Francone $^{1} \cdot$ Franco lafrate $^{1}$ (D) $\cdot$ Giorgio Maria Masci ${ }^{1} \cdot$ Simona Coco $^{1} \cdot$ Francesco Cilia $^{1} \cdot$ Lucia Manganaro $^{1}$. \\ Valeria Panebianco ${ }^{1}$. Chiara Andreoli ${ }^{2}$. Maria Chiara Colaiacomo ${ }^{2} \cdot$ Maria Antonella Zingaropoli $^{3}$. \\ Maria Rosa Ciardi ${ }^{3}$. Claudio Maria Mastroianni ${ }^{3}$. Francesco Pugliese ${ }^{4} \cdot$ Francesco Alessandri $^{4} \cdot$ Ombretta Turriziani $^{5}$. \\ Paolo Ricci ${ }^{1,2} \cdot$ Carlo Catalano $^{1}$
}

Received: 7 April 2020 / Revised: 5 June 2020 / Accepted: 12 June 2020 / Published online: 4 July 2020

(C) European Society of Radiology 2020

\begin{abstract}
Objectives To correlate a CT-based semi-quantitative score of pulmonary involvement in COVID-19 pneumonia with clinical staging of disease and laboratory findings. We also aimed to investigate whether CT findings may be predictive of patients' outcome. Methods From March 6 to March 22, 2020, 130 symptomatic SARS-CoV-2 patients were enrolled for this single-center analysis and chest CT examinations were retrospectively evaluated. A semi-quantitative CT score was calculated based on the extent of lobar involvement $(0: 0 \% ; 1,<5 \% ; 2: 5-25 \% ; 3: 26-50 \% ; 4: 51-75 \% ; 5,>75 \%$; range $0-5$; global score $0-25)$. Data were matched with clinical stages and laboratory findings. Survival curves and univariate and multivariate analyses were performed to evaluate the role of CT score as a predictor of patients' outcome.

Results Ground glass opacities were predominant in early-phase ( $\leq 7$ days since symptoms' onset), while crazy-paving pattern, consolidation, and fibrosis characterized late-phase disease ( $>7$ days). CT score was significantly higher in critical and severe than in mild stage $(p<0.0001)$, and among late-phase than early-phase patients $(p<0.0001)$. CT score was significantly correlated with CRP $(p<0.0001, r=0.6204)$ and D-dimer $(p<0.0001, r=0.6625)$ levels. A CT score of $\geq 18$ was associated with an increased mortality risk and was found to be predictive of death both in univariate (HR, 8.33; 95\% CI, 3.19-21.73; $p<0.0001$ ) and multivariate analysis (HR, 3.74; 95\% CI, 1.10-12.77; $p=0.0348$ ).

Conclusions Our preliminary data suggest the potential role of CT score for predicting the outcome of SARS-CoV-2 patients. CT score is highly correlated with laboratory findings and disease severity and might be beneficial to speed-up diagnostic workflow in symptomatic cases.

Key Points

- CT score is positively correlated with age, inflammatory biomarkers, severity of clinical categories, and disease phases.

- A CT score $\geq 18$ has shown to be highly predictive of patient's mortality in short-term follow-up.

- Our multivariate analysis demonstrated that CT parenchymal assessment may more accurately reflect short-term outcome, providing a direct visualization of anatomic injury compared with non-specific inflammatory biomarkers.
\end{abstract}

Keywords Severe acute respiratory syndrome coronavirus $2 \cdot$ COVID-19 $\cdot$ Pneumonia $\cdot$ Tomography, X-ray computed

Marco Francone and Franco Iafrate contributed equally to this work.

Franco Iafrate

francoiafrate@gmail.com

1 Department of Radiological, Oncological and Pathological Sciences, Policlinico Umberto I, Sapienza University of Rome, Viale Regina Elena 324, 00161 Rome, Italy

2 Unit of Emergency Radiology, Policlinico Umberto I, Policlinico Umberto I, Sapienza University of Rome, Viale del Policlinico 155, 00161 Rome, Italy
3 Department of Public Health and Infectious Diseases, Sapienza University of Rome, Piazzale Aldo Moro 5, 00185 Rome, Italy

4 Department of Anaesthesiology Critical Care Medicine and Pain Therapy, Policlinico Umberto I, Sapienza University, Viale Regina Elena 324, 00161 Rome, Italy

5 Department of Molecular Medicine, Sapienza University of Rome, Viale dell'Università 31, 00185 Rome, Italy 


$\begin{array}{ll}\text { Abbreviations } & \\ \text { ARDS } & \text { Acute respiratory distress syndrome } \\ \text { CDC } & \text { Center of Disease Control } \\ \text { COVID-19 } & \text { Coronavirus disease 2019 } \\ \text { CRP } & \text { C-reactive protein } \\ \text { GGO } & \text { Ground glass opacity } \\ \text { LLL } & \text { Left lower lobe } \\ \text { LUL } & \text { Left upper lobe } \\ \text { ML } & \text { Middle lobe } \\ \text { RLL } & \text { Right lower lobe } \\ \text { RT-PCR } & \text { Real-time polymerase chain reaction } \\ \text { RUL } & \text { Right upper lobe } \\ \text { SARS-CoV-2 } & \text { Severe acute respiratory syndrome } \\ & \text { coronavirus 2 } \\ \text { TAT } & \text { Turnaround time } \\ \text { WHO } & \text { World Health Organization }\end{array}$

Introduction

Severe acute respiratory syndrome coronavirus 2 (SARSCoV-2) or coronavirus disease 2019 (COVID-19), was firstly described in a series of 41 individuals presenting with undetermined forms of pneumonias in Wuhan, China, during December 2019 [1]. Since its first observation, SARS-CoV2 infection outbreak has transformed into an unprecedented worldwide healthcare emergency which recently reached the necessary epidemiological criteria to be declared pandemic by the World Health Organization [2]. The spread of the infection has been closely exponential in Italy which became, as of April 1, 2020, one of the world's centers of the outbreak together with the USA, with a total of 105,792 cases and the highest number of SARS-CoV-2 related deaths $(12,430)$ according to the latest World Health Organization (WHO) reports [3]. These numbers are unfortunately expected to increase as reported by Remuzzi et al in a recent modeling prediction published on The Lancet, despite the aggressive containment policy that has been imposed by the Italian government [4].

CT has a reported high sensitivity in patients infected by SARS-CoV-2, the reason why it is largely used to help patient management [5]. A high incidence of bilateral ground glass opacities (54\%) has been reported in a cohort of 82 asymptomatic carriers boarded on the international cruise ship "Diamond Princess." Those findings, observed in what temporarily became the largest cluster of SARS-CoV-2 cases outside China, potentially opened a major concern regarding a possible clinico-radiological dissociation in asymptomatic individuals, and its potential impact on clinical decision-making [6].

There is conversely, a growing evidence that sensitivity of combined nasal and pharyngeal swabs may be insufficient if obtained at a single time point, also depending on the technical characteristics of the test and method of specimen collection
$[7,8]$. The relatively long turnaround time (TAT) for viral testing together with the low sensitivity of a single real-time reverse-transcriptase polymerase-chain reaction (RT-PCR) assay of nasal and pharyngeal swab specimens also implies that a large number of SARS-CoV-2 patients would not be quickly identified and may not be appropriately managed.

We report our experience on a cohort of symptomatic patients who underwent chest $\mathrm{CT}$ following emergency room (ER) clinical triage.

In the current public health emergency, our hypothesis was that a highly sensitive test like CT would allow to speed-up diagnostic workflow and establish isolation at admission.

The aims of this retrospective study were to determine the correlation between a CT-based semi-quantitative score of pulmonary involvement with clinical staging of disease and to assess the role of $\mathrm{CT}$ in predicting short-term mortality.

\section{Materials and methods}

The present study was a single-center retrospective analysis conducted on an original cohort of 325 symptomatic patients with the suspicion of a SARS-CoV-2 interstitial pneumonia, who underwent chest CT scan in the Unit of Emergency Radiology of our hospital, from which patients with negative RT-PCR for SARS-CoV-2 were then excluded. The local ethical committee approved this retrospective study and written informed consent was waived.

\section{Clinical workflow and disease staging}

Routine blood tests and arterial blood gas (ABG) tests were performed for all patients and the following parameters were evaluated: C-reactive protein (CRP), D-dimer, lymphocyte count, and $\mathrm{PaO}_{2} / \mathrm{FiO}_{2}$ ratio. Vital parameters such as respiratory frequency and $\mathrm{O}_{2}$ saturation were also collected. In addition, all patients were followed during the observation period on their clinical evolution.

Clinical suspicion was established according to the Global surveillance for COVID-19 by the World Health Organization [9], when one of the following criteria was satisfied: patient with acute respiratory illness (fever and at least one sign/ symptom of respiratory disease, e.g., cough, shortness of breath), and a history of travel to or residence in a location reporting community transmission of COVID-19 disease during the 14 days prior to symptom onset; patient with any acute respiratory illness and having been in contact with a confirmed or probable COVID-19 case in the last 14 days prior to symptom onset; patient with severe acute respiratory illness (fever and at least one sign/symptom of respiratory disease, e.g., cough, shortness of breath; and requiring hospitalization) and in the absence of an alternative diagnosis that fully explains the clinical presentation. 
Disease severity score was evaluated in all cases, using the following criteria provided by the Chinese Center of Disease Control (CDC) [10]: mild disease including non-pneumonia or mild pneumonia (mild symptoms without dyspnea; respiratory frequency $<30 / \mathrm{min}$; blood oxygen saturation $\left(\mathrm{SpO}_{2}\right)$ $>93 \% ; \mathrm{PaO}_{2} / \mathrm{FiO}_{2}$ ratio $\geq 300 \mathrm{mmHg}$ ); severe disease including dyspnea, respiratory frequency $\geq 30 / \mathrm{min}, \mathrm{SpO}_{2} \leq 93 \%$, $\mathrm{PaO}_{2} / \mathrm{FiO}_{2}$ ratio $<300 \mathrm{mmHg}$, and/or lung infiltrates $>50 \%$ within 24 to $48 \mathrm{~h}$ (in our cohort, chest X-ray was never performed at admission and therefore, this last criterion was not applied in our study); critical disease including adult respiratory distress syndrome (ARDS) or respiratory failure, septic shock, and/or multiple organ dysfunction (MOD) or failure (MOF).

Depending on the timing of symptoms' onset, all cases were categorized as early (0-7 days) or late clinical manifestations (> 7 days) [11].

In all patients, nasopharyngeal swabs were collected, followed by RT-PCR assay to confirm the diagnosis. In the presence of an initial negative test, up to two additional RTPCRs were performed at intervals of 1 day or more (i.e., maximum 3 RT-PCR per patient within $72 \mathrm{~h}$ ).

The final population included only patients with a positive RT-PCR result for SARS-CoV-2.

\section{CT protocol}

Two multidetector CT scanners (Somatom Sensation 16 and Somatom Sensation 64; Siemens Healthineers) were used for all examinations.

Scanning parameters were identical to the manufacturer's standard recommended pre-setting for a thorax routine. Images were reconstructed with a 1-mm slice thickness in all cases using the classic filtered back-projection method with a soft tissue kernel of B20 and a lung kernel of B60. Coronal and sagittal multiplanar reconstructions were also available in all cases.

Implementation of appropriate infection prevention and control measures were arranged in all suspected CT cases, consisting of prompt sanitation of CT facility and patient's isolation.

\section{Image analysis}

Definitions of radiological terms like ground glass opacity (GGO), crazy-paving pattern, and pulmonary consolidation were based on the standard glossary for thoracic imaging reported by the Fleischner Society [12]. Based on previous publication [13, 14], diagnosis of a suspected SARS-CoV-2 pneumonia was established considering the following chest $\mathrm{CT}$ patterns: GGO, crazy-paving, and consolidation.

In all cases, a semi-quantitative CT severity scoring proposed by Pan et al [15] was calculated per each of the 5 lobes considering the extent of anatomic involvement, as follows: 0 , no involvement; $1,<5 \%$ involvement; $2,5-25 \%$ involvement; 3, 26-50\% involvement; 4, 51-75\% involvement; and $5,>75 \%$ involvement (Fig. 1). The resulting global CT score was the sum of each individual lobar score and (0 to 25). When present, related features such as fibrosis, subpleural lines, reversed "halo sign," pleural effusion, and lymphadenopathy were also described.

Distribution of lung abnormalities was also classified as predominately peripheral, central, or both peripheral and central, in each case analyzed. Lung parenchyma was, additionally, anatomically divided into the anterior and posterior zone by drawing a vertical line through the midpoint of the diaphragm in the sagittal reconstruction [11].

\section{Statistical analysis}

Data were analyzed using statistical software (Prism version 8.3, GraphPad Software). Continuous variables were expressed as mean value \pm standard deviation (SD). The Mann-Whitney test was used for single comparisons, while the Kruskal-Wallis test was used for multiple comparisons. The frequencies of demographic and clinical characteristics of populations were expressed as the number (percentage) of occurrences and were compared using the 2-tailed $\chi^{2}$ test or Fisher's exact test, so as for the frequencies of $\mathrm{CT}$ features in early- versus late-phase and for comparisons between mortality rate both versus age ranges and clinical stages. Pearson correlation test was used for correlations between CT score versus laboratory findings.

The Kaplan-Meier method was used to evaluate the relationship between CT score and all-cause mortality, which were compared with the log-rank test. To determine the optimal cut-off point for CT score as all-cause mortality predictor, we used the Cox proportional hazards regression modeling.

Univariate analysis between mortality and other variables including sex, age, CT score, CRP, and D-dimer levels was also performed and statistically significant variables were used as independent variables in multivariate analysis to identify independent predictors of death in COVID-19 patients. A $p$ value $<0.05$ was considered to be statistically significant.

\section{Results}

\section{Population, clinical presentation, and laboratory findings}

Starting from March 6 up to March 22, 2020, a total of 1274 patients underwent a nasopharyngeal swab followed by one up to three RT-PCR assays in our Institution. The mean turnaround time (TAT) for RT-PCR results was $7.8 \pm 3.9 \mathrm{~h}$. The mean time for chest $\mathrm{CT}$ reporting was $11.2 \pm 3.6 \mathrm{~min}$. CT scanner's sanitation required approximately $30 \mathrm{~min}$. 
Fig. 1 Different CT score of RLL involvement in COVID-19

pneumonia on axial, sagittal, and coronal images. $0 \%$ of RLL lobe involvement (a); $<5 \%$ of RLL

involvement (b); $20 \%$ of RLL involvement (c); $40 \%$ of RLL lobe involvement (d); $70 \%$ of RLL involvement (e); $>75 \%$ of RLL involvement (f)

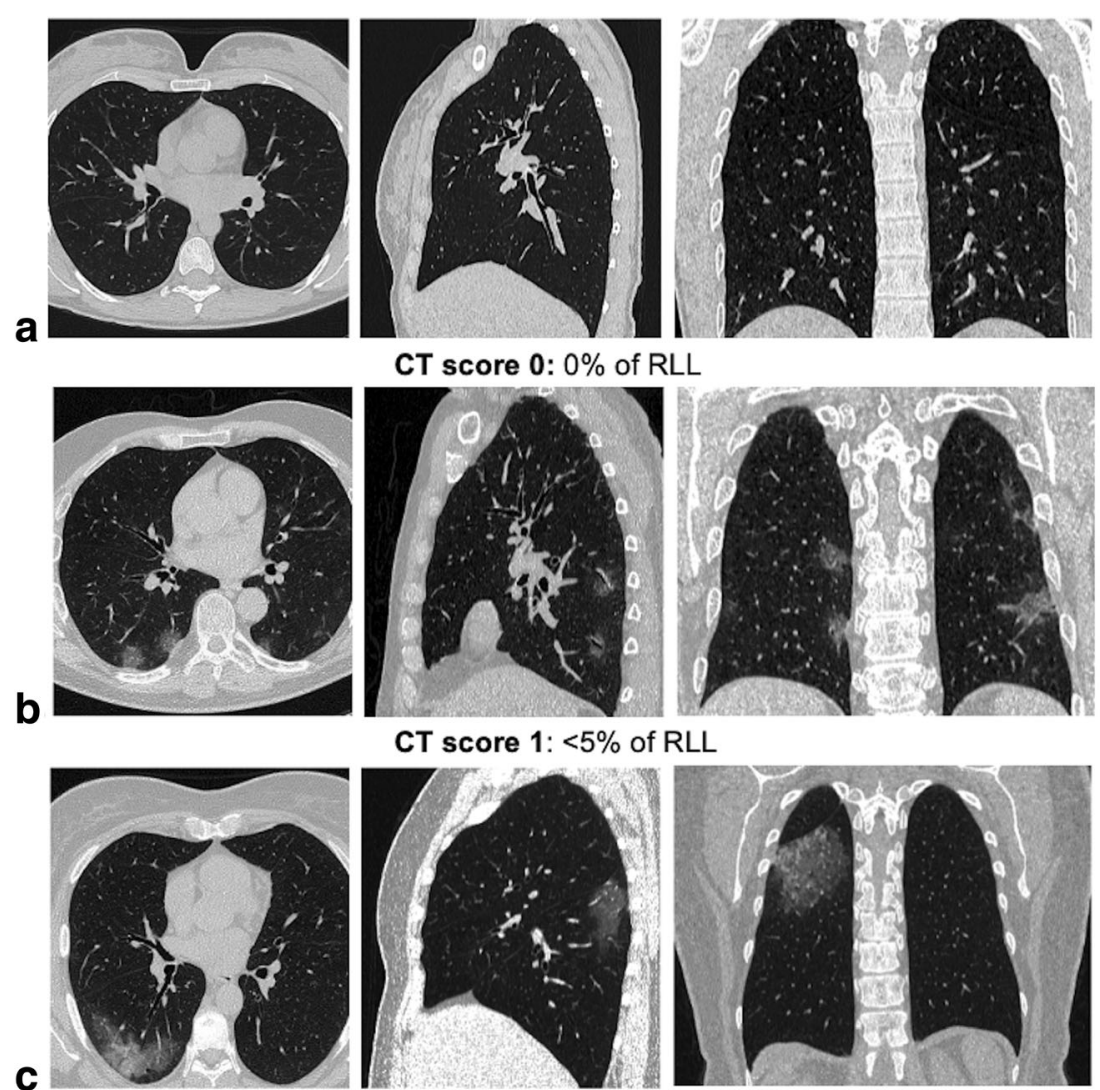

CT score 2: $5-25 \%$ of RLL
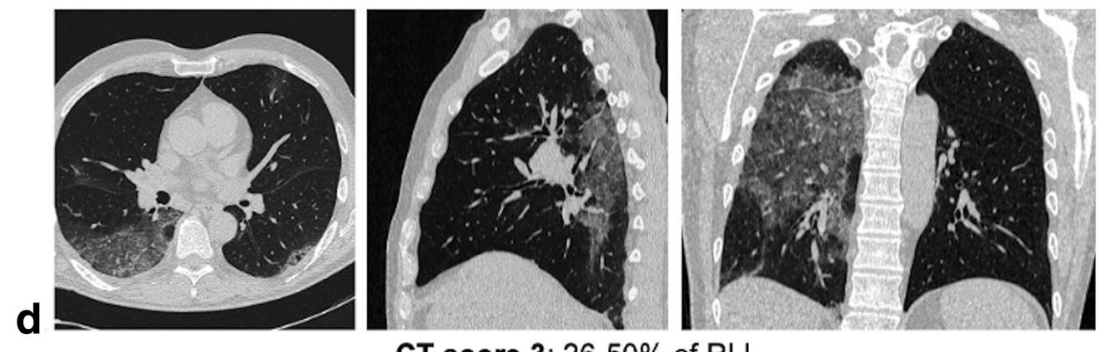

CT score 3: $26-50 \%$ of RLL
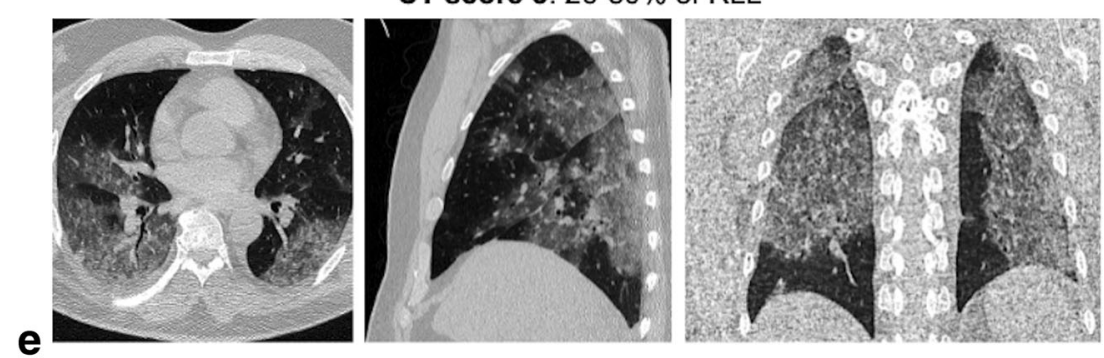

CT score $4: 51-75 \%$ of RLL
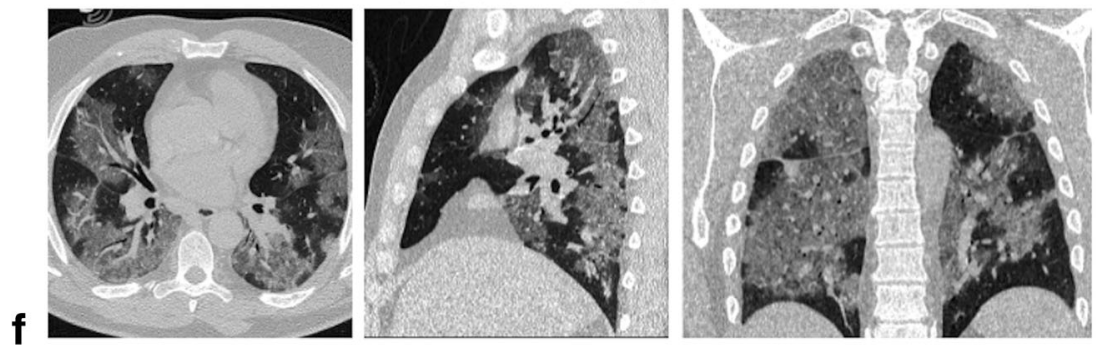

CT score 5 : > 75\% of RLL 
From the original cohort of 325 cases with the suspicion of COVID-19 infection, final population included 130 patients (84 males, 46 females; mean age $63.2 \pm 15.8$, range $27-$ 90 years) with a positive RT-PCR test for SARS-CoV-2. One-hundred and twenty-six patients had a positive diagnosis on first RT-PCR, 3 patients on the second RT-PCR test and in 1 patient after three tests. Among these, 7 patients were discharged and self-isolated at home after $\mathrm{CT}$ was performed.

The most common clinical manifestations were fever, coughing, and dyspnea. Increased CRP levels (CRP $>0.5 \mathrm{mg} / \mathrm{dL}$ ) were found in 113/130 (86.9\%) patients with a mean value of $8.3 \mathrm{mg} / \mathrm{dL} \pm 11.1$ and increased D-dimer levels $(>500 \mathrm{ng} / \mathrm{mL}$ ) were found in $114 / 130(87.7 \%)$ patients with a mean value of $1767 \mathrm{ng} / \mathrm{mL} \pm 1425$.

Decreased lymphocyte count was observed in $80 / 130$ $(61.5 \%)$ patients, decreased $\mathrm{O}_{2}$ saturation in $53 / 130(40.1 \%)$, and decreased $\mathrm{PaO}_{2} / \mathrm{FiO}_{2}$ ratio in $86 / 130(66.2 \%)$ patients.

Demographic and clinical characteristics of the two populations are summarized in Table 1.

Based on the Chinese CDC clinical scoring for SARSCoV-2 infection [10], seventy-nine $(60.8 \%)$ were classified as mild, $42(32.3 \%)$ as severe, and 9 patients $(6.9 \%)$ as critical.

\section{CT features and disease scoring}

The most common patterns of disease included GGO, observed in 125 patients $(96.2 \%)$, followed by crazy-paving pattern $(n=68 ; 52.3 \%)$ and parenchymal consolidations $(n=75$; $57.7 \%$ ) (Fig. 2). Related CT features were found as follows: fibrosis $(n=53 ; 40.8 \%)$, subpleural lines $(n=28 ; 21.5 \%)$, reversed "halo sign" $(n=5 ; 3.8)$, pleural effusion $(n=6 ; 13 \%)$, and lymphadenopathy $(n=20 ; 6.2 \%)$. Lobar involvement, lesion distribution, and disease localization in the pulmonary parenchyma were also observed. Pathological involvement was most common in the inferior lobes, right lower lobe (RLL) in 122 patients (93.8\%), and left lower lobe (LLL) in 123 patients $(94.6 \%)$. The mean CT scores were found as follows: $2.2 \pm 1.5$ for the right upper lobe (RUL), $1.8 \pm 1.5$ for the middle lobe (ML), $3.1 \pm 1.3$ for the right lower lobe (RLL), $2.2 \pm 1.2$ for the left upper lobe (LUL), and $3 \pm 1.4$ for the left lower lobe (LLL) (Table 2 and Fig. 3). The mean global CT score was $12.3 \pm 11.1$. Only one patient did not show any parenchymal involvement at CT and was therefore scored as 0 . Comparisons have been made between lobes for each lung. Regarding the right lung, mean CT score was significantly higher in RLL than in ML $(p<0.0001)$ and RUL $(p<0.0001)$; no significant difference was found between RUL and ML ( $p=0692)$. Concerning the left lung, mean CT score was significantly higher in LLL than in LUL $(p<0.0001)$ (Table 2 and Fig. 3).

Regarding distribution of parenchymal abnormalities on sagittal reconstructions, pathological findings were posterior in 67 patients (51.5\%) and anterior in 4 patients (3.1\%). In the
Table 1 Demographic and clinical characterstics of our study population

\begin{tabular}{lc}
\hline Characteristic & SARS-CoV-2+ patients \\
\hline Sex, no. of patients/total patients $(\%)$ & \\
Male & $84 / 130(64.6 \%)$ \\
Female & $46 / 130(35.4 \%)$ \\
Age range, no. of patients/total patients $(\%)$ & \\
$0-25$ & $0 / 130(0 \%)$ \\
$26-50$ & $29 / 130(22.3 \%)$ \\
$51-75$ & $65 / 130(50 \%)$ \\
$>75$ & $36 / 130(27.7 \%)$ \\
Symptoms, no. of patients/total patients $(\%)$ & \\
Fever & $113 / 130(86.9 \%)$ \\
Coughing & $67 / 130(51.5 \%)$ \\
Dyspnea & $56 / 130(43.1 \%)$ \\
Diarrhea & $12 / 130(9.2 \%)$ \\
Headache & $4 / 130(3.1 \%)$ \\
Clinical and laboratory findings, no. & \\
of patients/total patients (\%) & \\
Increased CRP level & $113 / 130(86.9 \%)$ \\
Increased D-dimer level & $114 / 130(87.7 \%)$ \\
Leukopenia & $39 / 130(30 \%)$ \\
Decreased lymphocyte count & $80 / 130(61.5 \%)$ \\
Decreased $\mathrm{O}_{2}$ saturation & $53 / 130(40.1 \%)$ \\
Decreased PaO ${ }_{2} /$ FiO ${ }_{2}$ ratio & $86 / 130(66.2 \%)$ \\
Comorbidities, no. of patients & \\
Hypertension & 42 \\
Obesity & 17 \\
Diabetes & 15 \\
Chronic obstructive pulmonary disease & 9 \\
Neoplasm & 8 \\
Chronic kidney disease & \\
\hline & \\
\hline & \\
\hline
\end{tabular}

remaining 58 cases (44.6\%), involvement of both anterior and posterior areas was observed. Fifty-six patients $(43.1 \%)$ were found to have peripheral involvement and 73 patients $(56.1 \%)$ presented both peripheral and central pattern distribution. In 1 $(0.08 \%)$ patient, no parenchymal involvement was found (Table 2).

\section{CT patterns in early versus late-phase disease}

Forty-six out of 130 patients (35.4\%) were classified to have an early-phase disease and 84/130 patients (64.6\%) to have a late-phase disease. GGO pattern was significantly prevalent in early-phase disease (34 patients; $73.9 \% ; p<0.0001$ ) compared with late-phase disease ( $n=28$ patients; $33.3 \%$ ), while crazy-paving and consolidation patterns were significantly more common in late-phase. Regarding CT-related features, subpleural lines were significantly prevalent in early-phase, while fibrosis in late-phase (Table 3). No significant 

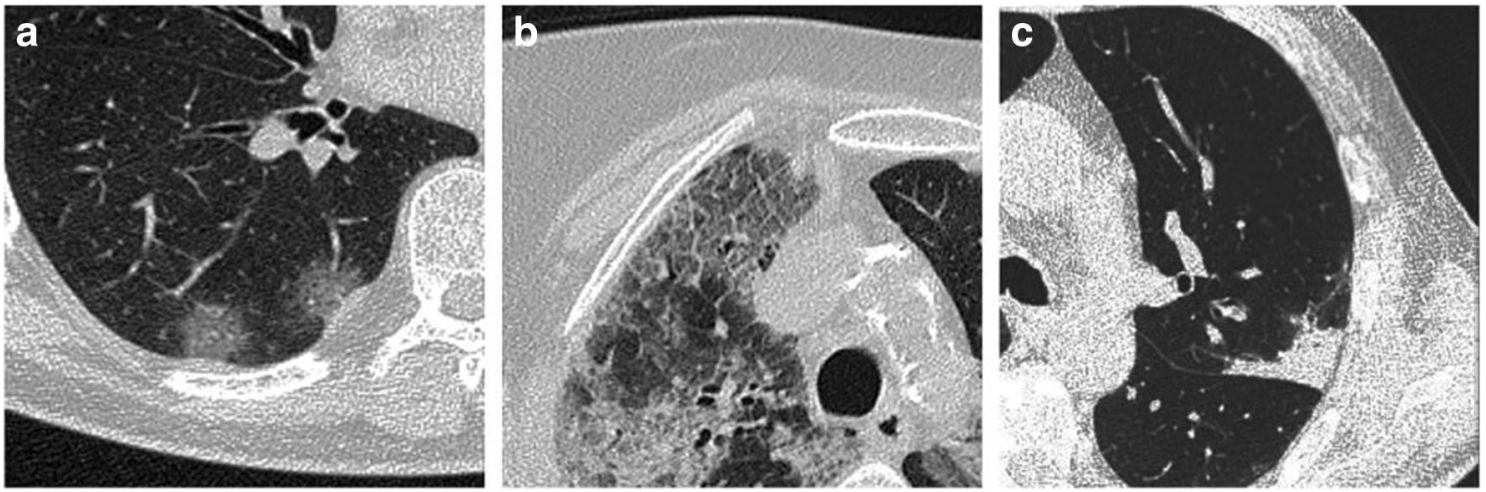

Fig. 2 Chest CT findings of COVID-19 pneumonia on axial images. GGO (a); crazy-paving pattern (GGO with superimposed inter- and intralobular septal thickening) (b); consolidation (c)

differences were found for reversed "halo sign," pleural effusion and lymphadenopathy between early- and late-phase.

\section{Correlations between CT score and laboratory findings}

CT score was compared with clinical categories and significant difference was observed when all categories were com- pared together $(p<0.0001)$. When multiple comparisons were made, CT score was significantly higher in the critical category (mean value \pm SD: $20.3 \pm 3$; range $15-24$ ) than in the mild category $(8.7 \pm 4$; range $0-19)(p<0.0001)$. CT score was also significantly higher in the severe category $(17.4 \pm 3.1$; range $11-24)$ versus the mild category $(8.7 \pm 4$; range $0-19)$ $(p<0.0001)$. No statistical significance was observed between severe and critical categories $(p=0.7921)$ (Fig. 4).
Table 2 Frequency of involvement of each lobe with related CT score, disease localization, and main patterns and features in SARS-CoV-2+ patients

\begin{tabular}{|c|c|c|c|}
\hline Categories & No. $(\%)$ of SARS-CoV-2+ patients & $\mathrm{CT}$ mean score $\pm \mathrm{SD}$ & $p$ value \\
\hline \multicolumn{4}{|l|}{ Lung Lobe } \\
\hline Right upper lobe (RUL) & $107(82.3)$ & $2.2 \pm 1.5$ & \multirow{3}{*}{$\begin{array}{l}<0.0001 \\
]<0.0001\end{array}$} \\
\hline Middle lobe (ML) & $102(78.4)$ & $1.8 \pm 1.5$ & \\
\hline Right lower lobe (RLL) & $122(93.8)$ & $3.1 \pm 1.3$ & \\
\hline Left upper lobe (LUL) & $113(86.9)$ & $2.2 \pm 1.2$ & \multirow{2}{*}{]$<0.0001$} \\
\hline Left lower lobe (LLL) & $123(94.6)$ & $3 \pm 1.4$ & \\
\hline \multicolumn{4}{|l|}{ Distribution } \\
\hline Peripheral & $56 / 130(43.1 \%)$ & & \\
\hline Peripheral and central & $73 / 130(56.1 \%)$ & & \\
\hline None & $1 / 130(0.8 \%)$ & & \\
\hline \multicolumn{4}{|l|}{ Lung area } \\
\hline Anterior & $4 / 130(3.1 \%)$ & & \\
\hline Posterior & $67 / 130(51.5 \%)$ & & \\
\hline Anterior and posterior & $58 / 130(44.6 \%)$ & & \\
\hline None & $1 / 130(0.8 \%)$ & & \\
\hline \multicolumn{4}{|l|}{ Main pattern } \\
\hline Ground glass opacity & $62 / 130(47.7 \%)$ & & \\
\hline Crazy paving & $41 / 130(31.5 \%)$ & & \\
\hline Consolidation & $26 / 130(20 \%)$ & & \\
\hline No patterns & $1 / 130(0.8 \%)$ & & \\
\hline \multicolumn{4}{|l|}{ Related features } \\
\hline Fibrosis & $53 / 130(40.8 \%)$ & & \\
\hline Subpleural lines & $28 / 130(21.5 \%)$ & & \\
\hline Reversed "halo sign" & $5 / 130(3.8 \%)$ & & \\
\hline Pleural effusion & $6 / 130(13 \%)$ & & \\
\hline Lymphadenopathy & $20 / 130(6.2 \%)$ & & \\
\hline
\end{tabular}




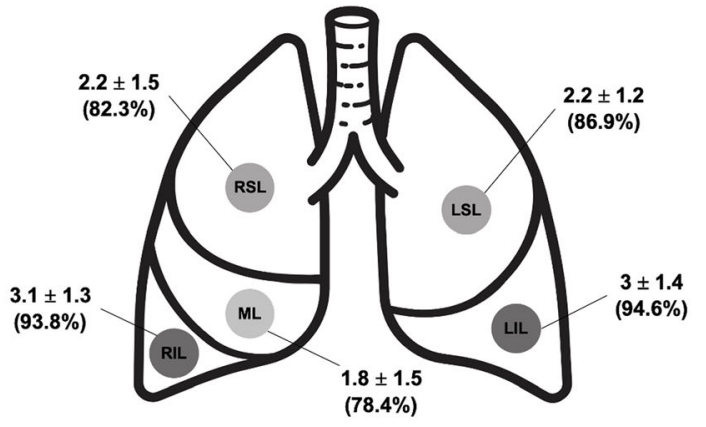

a

Fig. 3 Lobar CT scores (a) and CT score comparisons between lobes in right and left lungs (b) in SARS-CoV-2+ patients. Data are expressed as mean value \pm SD (\% of occurrences of involvement for each lobe) (a).

When compared with disease phase, CT score was found to be significantly higher among late-phase than in early-phase patients $(p<0.0001)$ (Fig. 4).

Statistically significant correlations were found between CT score vs CRP $(p<0.0001, r=0.6204)$ and D-dimer $(p<0.0001, r=0.6625)$ levels.

No statistically significant correlation was observed between CT score and lymphocyte count $(p=0.0538, r=-0.1730)$.

CT score was finally compared between age range groups, and a statistically significant difference was found when all groups were compared together $(p=0.0018)$. When multiple comparisons were made, CT score was significantly higher in age range $>75$ than in age range 26-50 $(p=0.0012)$. CT score was also significantly higher in age range $51-75$ than in age range $26-50(p=0.0367)$. No statistical significance was observed in group 51-75 versus $>75$ years old patients $(p=0.3605)$.

\section{Kaplan-Meier survival curves and univariate and multivariate analyses}

Out of the 130 patients in the study, 20 (15.4\%) died during a mean follow-up of $14.2 \pm 4.4$ days (range 1-24 days), 16 of
Left Lung

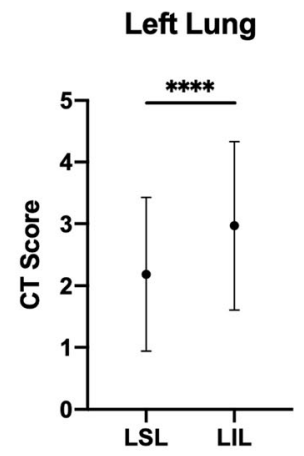

b

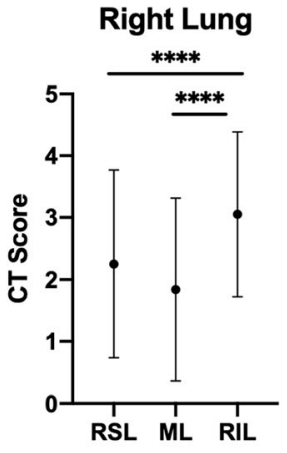

Black dots express mean value, branches express SD $(* * * * p<0.0001)$ (b). RUL, right upper lobe; ML, middle lobe; RLL, right lower lobe; LUL, left upper lobe; LLL, left lower lobe

which presented at least one of the previously mentioned comorbidities. Hypertension was reported in $8 / 20$ of deaths (40\%), while no significant comorbidities were present in $4 /$ 20 cases $(20 \%)$.

All-cause mortality was significantly higher in patients $\geq 75$ years old $(n=12 / 36 ; 33.3 \%)$ than in patients $<75$ years old $(n=8 / 94 ; 8.5 \%)(p=0.0083)$.

The mortality rate was also significantly higher among critical patients $(9 / 9 ; 100 \%)$ compared with mild $(3 / 79 ; 3.8 \%)$ and severe $(8 / 42 ; 19 \%)$ (respectively, $p<0.0001$ and $p=$ $0.0091)$. It was also significantly higher in severe than in mild patients $(p=0.0204)$.

According to the Kaplan-Meier analysis, the risk of death significantly increased with the increase of CT score value using an estimated cut-off of $\geq 18$ (log-rank $p<0.0001$; hazard ratio [HR], $0.098 ; p=0.0201$ ) on a 24-day follow-up period (Fig. 5).

Univariate analysis showed a significant higher risk of death in patients with a CT score $\geq 18$ (HR, 8.33; 95\% CI, $3.19-21.73 ; p<0.0001)$. Also risk of death was significantly correlated with increase of age (HR, 1.07; 95\% CI, 1.03-1.11; $p=0.0014)$, CRP (HR, 1.06; 95\% CI, 1.03-1.09; $p<0.0001$ )

Table 3 CT features of SARS$\mathrm{CoV}-2+$ patients and differences between early and late disease phase

\begin{tabular}{lccr}
\hline CT features in SARS-CoV-2+ patients & Early-phase, $n=46$ & Late-phase, $n=84$ & $p$ value \\
\hline Main CT pattern & & & \\
Ground glass opacity & $34 / 46(73.9 \%)$ & $28 / 84(33.3 \%)$ & $<0.0001$ \\
Crazy paving & $7 / 46(15.2 \%)$ & $34 / 84(40.5 \%)$ & 0.0031 \\
Consolidation & $4 / 46(8.7 \%)$ & $22 / 84(26.2 \%)$ & 0.0211 \\
No patterns & $1 / 46(2.2 \%)$ & $0 / 84(0 \%)$ & 0.3538 \\
Related features & & & \\
Fibrosis & $8 / 46(17.4 \%)$ & $45 / 84(53.6 \%)$ & $<0.0001$ \\
Subpleural lines & $15 / 46(32.6 \%)$ & $13 / 84(15.5 \%)$ & 0.0275 \\
Reversed "halo sign" & $1 / 46(2.2 \%)$ & $4 / 84(4.8 \%)$ & 0.6565 \\
Pleural effusion & $6 / 46(13 \%)$ & $14 / 84(16.7 \%)$ & 0.7999 \\
Lymphadenopathy & $2 / 46(4.3 \%)$ & $6 / 84(7.1 \%)$ & 0.7115 \\
\hline
\end{tabular}


Fig. 4 Comparisons between CT scores versus clinical categories (a) and disease phases (b) in SARS-CoV-2+ patients. Larger horizontal lines express mean values, shorter lines express $\mathrm{SD}$ $(* * * * p<0.0001)$
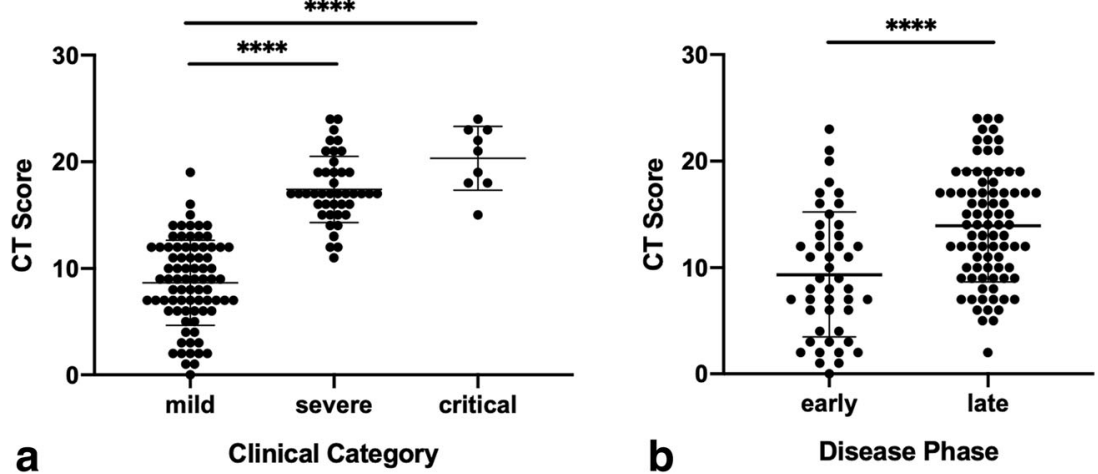

and D-dimer levels (HR, 1.001; 95\% CI, 1-1.001; $p=0.0001$ ). No statistical significance was found considering sex. Multivariate analysis conducted on variables showing statistical significance in univariate analysis confirmed the role of CT score as an independent predictor of death (HR, 3.74; 95\% CI, 1.10-12.77; $p=0.0348)$ together with age (HR, 1.07; $95 \%$ CI, $1.02-1.12 ; p=0.0045$ ). Area under the curve (AUC) for multivariate model was 0.762 (95\% CI 0.647-0.877).

\section{Discussion}

The main hallmark of COVID-19 pneumonia, as confirmed in our study, is the presence of bilateral GGOs with or without consolidative areas, with a predominant peripheral, lower lobes, and posterior anatomic distribution $[11,16]$.

The prevalence of GGOs observed in early phases of the disease in our patient's series likely represents the imaging correlate of the acute-phase diffuse alveolar damage that has reported, with airspace edema, bronchiolar fibrin, and interstitial thickening [17]. Late disease progresses with the activation of humoral and cellular immunity mediated by virusspecific $\mathrm{B}$ and $\mathrm{T}$ cells, causing an intense production of proinflammatory cytokines that may trigger an uncontrolled autoimmune reaction. These findings may explain the higher

\section{Kaplan-Meier curve}

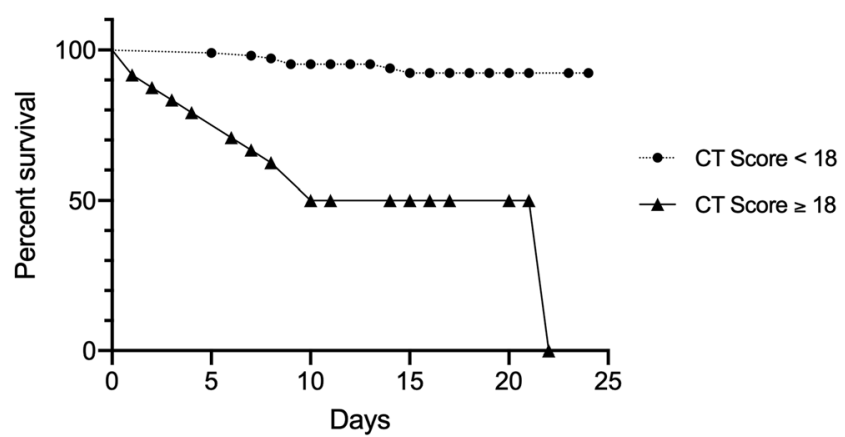

Fig. 5 Kaplan-Meier survival curve. Estimated survival rate comparison between SARS-CoV-2+ patients with CT score $<18$ and $\geq 18$. Percentage of survival is expressed on the $y$-axis, while time (days) of the observation period is expressed on the $x$-axis prevalence of crazy-paving pattern and consolidation areas that we have observed in our late disease population, which probably refer to a combination of alveolar edema, bacterial superinfection, and interstitial inflammatory changes [18].

Clinical course of the disease is unpredictable, due to the heterogeneity of its manifestations ranging from asymptomatic and/or subclinical forms to critical disease with ARDS or multiorgan failure.

There is no currently available prognostic biomarker to identify patients requiring immediate medical attention and to estimate their associated mortality rate [19].

Our hypothesis was that CT prediction of disease progression and its correlation with clinical-laboratory findings may be helpful to assist medical staff in triaging patients and to timely establish symptomatic treatment, although COVID-19 therapy is still based on merely empirical decisions rather than on the evidence of large clinical trials [20].

To verify this assumption, we used a previously validated CT score, based on the lobar extent of disease as reported by Pan et al [15].

A Kaplan-Meier survival analysis was constructed on the basis of CT score, to confirm prognostic significance of chest CT findings over an observational period of 24 days. Using this method, we were able to demonstrate that a cut-off value of 18 is highly predictive of short-term mortality (Fig. 5). Similar observations were recently reported by Colombi et al [21], who found a positive correlation between the extent of CT lung involvement and intensive care unit admission or death, in a cohort of 236 patients.

A different scoring system was proposed in literature either in COVID-19 and H7N9 pneumonia [22, 23], combining the extent of pulmonary involvement with specific attenuation patterns (i.e., normal, ground-glass, and consolidation). Using this method, a final cumulative score ranging from 0 to 72 could be obtained, which yielded a sensitivity of $85.6 \%$ and a specificity of $84.5 \%$ for the prediction of mortality in a population of 27 SARS-CoV-2 patients [22].

CT scoring was also compared with most important independent risk factors associated with ARDS and fatal outcome, which were reported to be age, dyspnea at admission, and the presence of pre-existing comorbidities like coronary arteries 
and cerebrovascular diseases [24, 25]. Our mortality data have confirmed the prominent prognostic importance of age: allcause mortality was significantly higher in patients older than 75 years $(n=12 / 36 ; 33.3 \%)$. Age-dependent mortality was also demonstrated in our univariate analysis, showing an increasing risk of death of 1.069 times per year-increase.

Serum levels of CRP and D-dimer were similarly found to be commonly increased in COVID-19 patients and strongly associated with outcome, respectively as a consequence of the diffuse inflammatory activation and disseminated coagulopathy characterizing severe forms of disease [26, 27]. These observations have been confirmed by our univariate analysis, showing a statistical significance of both PCR and D-dimer as mortality determinants.

However, when including all predictors in our multivariate model, only CT score and age remained significant compared with CRP and D-dimer. These results substantially validate our hypothesis that $\mathrm{CT}$ parenchymal assessment may more accurately reflect short-term outcome, providing a direct visualization of anatomic injury compared with non-specific inflammatory biomarkers.

Using the clinical criteria provided by the Chinese CDC, we also aimed to correlate disease severity with CT findings. As expected, CT scores were significantly lower in the mild category than in the severe and critical categories, confirming high correlation between imaging findings and clinical stages.

The diagnostic role of chest CT remains controversial and debated in the scientific community. While several authors and radiological societies do not recommend the use of $\mathrm{CT}$ as a firstline test $[28,29]$, our study seems to suggest that a highly sensitive imaging method like $\mathrm{CT}$, although not as specific, might be beneficial to speed up diagnostic and therapeutic workflow. On the basis of the experience from Orsi et al [30], CT could be used to discharge patients with negative imaging results and clinical stability, without waiting for the results of the swab test, particularly in the presence of negative/inconclusive radiographic findings or a possible false-negative result.

Notably, we found a statistically significant difference in $\mathrm{CT}$ reporting time vs. RT-PCR results TAT (mean time respectively $11.2 \mathrm{~min}$ versus $7.8 \mathrm{~h}$ ), likely as a consequence of an exceptionally increased clinical workload for our clinical lab. Viral testing had to be repeated up to three times in 4 individuals, thus prolonging definition of a final diagnosis of SARS-CoV-2 up to 3 days after hospital's admission. On the other hand, CT examinations had to be interspersed by sanitation process between one patient and another; however, this process did not interfere with diagnostic workflow, having available two $\mathrm{CT}$ scanners.

We could assume that chest CT can supplement part of the known limitations of RT-PCR assay, which has shown to be a limited sensitivity test, especially when performed on swabs instead of sputum [8], and requires a relatively long turnaround time to get a final diagnosis.
Our study has some limitations. We performed a retrospective analysis evaluation in a relatively limited cohort of patients, but the severity of the current healthcare emergency implies that a prospective evaluation would have been extremely complex and longer to complete.

Our survival analysis also lacks longer follow-up data, being only limited to a relatively short observational period (24 days) of our patient's cohort. Clinical course of viral pneumonias is, however, expected to be limited to a maximum of 4 weeks in most of the cases, meaning that the majority of events would be expected to occur within this temporal window.

Future larger studies could supplement with additional information the significance of CT in the diagnostic workflow of SARS-CoV-2+ patients. For similar reasons, direct impact of CT on clinical decision making has not been assessed.

\section{Conclusion}

CT scoring could help to stratify patient's risk and predict short-term outcome of patients with COVID-19 pneumonia.

The extent of CT damage is highly correlated with various parameters of disease, including clinical staging and laboratory parameters.

Finally, our study strongly supports the use of chest CT in patients with COVID-19 pneumonia, which could be used as a rapid and effective gatekeeper to rule-out patients with a low likelihood of disease.

Future larger studies are expected to better clarify its impact on clinical decision-making, waiting for larger clinical trials.

Acknowledgments We would like to acknowledge all the colleagues of Unit of Emergency Radiology, Department of Radiological Sciences and Department of Public Health and Infectious diseases for their great effort in patients' management.

Funding information The authors state that this work has not received any funding.

\section{Compliance with ethical standards}

Guarantor The scientific guarantor of this publication is Franco Iafrate.

Conflict of interest The authors of this manuscript declare no relationships with any companies whose products or services may be related to the subject matter of the article.

Statistics and biometry Maria Antonella Zingaropoli kindly provided statistical advice for this manuscript.

Informed consent Written informed consent was waived due to the retrospective nature of the study.

Ethical approval This study was approved by the local ethic committee. 


\section{Methodology}

- retrospective

- diagnostic or prognostic study

- performed at one institution

\section{References}

1. Huang C, Wang Y, Li X et al (2020) Clinical features of patients infected with 2019 novel coronavirus in Wuhan, China. Lancet 395:497-506. https://doi.org/10.1016/S0140-6736(20)30183-5

2. WHO Director-General's opening remarks at the media briefing on COVID-19 - 11 March 2020. https://www.who.int/dg/speeches/ detail/who-director-general-s-opening-remarks-at-the-mediabriefing-on-covid-19\%2D\%2D-11-march-2020. Accessed 27 Mar 2020

3. Coronavirus disease (COVID-19) Situation Dashboard. https:// experience.arcgis.com/experience/685d0ace $521648 \mathrm{f} 8 \mathrm{a}$ 5 beeeee1b9125cd. Accessed 2 Apr 2020

4. Remuzzi A, Remuzzi G (2020) COVID-19 and Italy: what next? Lancet 0: https://doi.org/10.1016/S0140-6736(20)30627-9

5. Ai T, Yang Z, Hou H et al (2020) Correlation of chest CT and RTPCR testing in coronavirus disease 2019 (COVID-19) in China: a report of 1014 cases. Radiology 200642. https://doi.org/10.1148/ radiol.2020200642

6. Inui S, Fujikawa A, Jitsu M et al (2020) Chest $\mathrm{CT}$ findings in cases from the cruise ship "diamond princess" with coronavirus disease 2019 (COVID-19). Radiology: Cardiothoracic Imaging 2:e200110. https://doi.org/10.1148/ryct.2020200110

7. Wang W, Xu Y, Gao R et al (2020) Detection of SARS-CoV-2 in different types of clinical specimens. JAMA. https://doi.org/10. 1001/jama.2020.3786

8. Han H, Luo Q, Mo F, Long L, Zheng W (2020) SARS-CoV-2 RNA more readily detected in induced sputum than in throat swabs of convalescent COVID-19 patients. Lancet Infect Dis 0. https:// doi.org/10.1016/S1473-3099(20)30174-2

9. World Health Organization (2020) Global surveillance for COVID19 caused by human infection with COVID-19 virus: interim guidance, 20 march 2020. World Health Organization

10. Wu Z, McGoogan JM (2020) Characteristics of and important lessons from the coronavirus disease 2019 (COVID-19) outbreak in China: summary of a report of 72314 cases from the Chinese Center for Disease Control and Prevention. JAMA 323:12391242. https://doi.org/10.1001/jama.2020.2648

11. Zhou S, Wang Y, Zhu T, Xia L (2020) CT features of coronavirus disease 2019 (COVID-19) pneumonia in 62 patients in Wuhan, China. AJR Am J Roentgenol:1-8. https://doi.org/10.2214/AJR. 20.22975

12. Hansell DM, Bankier AA, MacMahon H, McLoud TC, Müller NL, Remy J (2008) Fleischner society: glossary of terms for thoracic imaging. Radiology 246:697-722. https://doi.org/10.1148/radiol. 2462070712

13. Salehi S, Abedi A, Balakrishnan S, Gholamrezanezhad A (2020) Coronavirus disease 2019 (COVID-19): a systematic review of imaging findings in 919 patients. AJR Am J Roentgenol:1-7. https:// doi.org/10.2214/AJR.20.23034

14. Ye Z, Zhang Y, Wang Y, Huang Z, Song B (2020) Chest CT manifestations of new coronavirus disease 2019 (COVID-19): a pictorial review. Eur Radiol. https://doi.org/10.1007/s00330-02006801-0

15. Pan F, Ye T, Sun P et al (2020) Time course of lung changes on chest CT during recovery from 2019 novel coronavirus (COVID-
19) pneumonia. Radiology 200370. https://doi.org/10.1148/radiol. 2020200370

16. Simpson S, Kay FU, Abbara S et al (2020) Radiological Society of North America expert consensus statement on reporting chest CT findings related to COVID-19. Endorsed by the Society of Thoracic Radiology, the American College of Radiology, and RSNA. Radiology: Cardiothoracic Imaging 2:e200152. https://doi.org/10. 1148/ryct.2020200152

17. Li X, Geng M, Peng Y, Meng L, Lu S (2020) Molecular immune pathogenesis and diagnosis of COVID-19. J Pharm Anal. https:// doi.org/10.1016/j.jpha.2020.03.001

18. Tian S, Xiong Y, Liu H et al (2020) Pathological study of the 2019 novel coronavirus disease (COVID-19) through postmortem core biopsies. Mod Pathol:1-8. https://doi.org/10.1038/s41379-0200536-x

19. Yan L, Zhang H-T, Goncalves J et al (2020) An interpretable mortality prediction model for COVID-19 patients. Nat Mach Intell:16. https://doi.org/10.1038/s42256-020-0180-7

20. Rubin EJ, Harrington DP, Hogan JW, et al (2020) The urgency of care during the Covid-19 pandemic - learning as we go. N Engl J Med 0:null. https://doi.org/10.1056/NEJMe2015903

21. Colombi D, Bodini FC, Petrini M et al (2020) Well-aerated lung on admitting chest $\mathrm{CT}$ to predict adverse outcome in COVID-19 pneumonia. Radiology:201433. https://doi.org/10.1148/radiol. 2020201433

22. Yuan M, Yin W, Tao Z, Tan W, Hu Y (2020) Association of radiologic findings with mortality of patients infected with 2019 novel coronavirus in Wuhan, China. PLoS One 15:e0230548. https://doi.org/10.1371/journal.pone.0230548

23. Feng F, Jiang Y, Yuan M et al (2014) Association of radiologic findings with mortality in patients with avian influenza H7N9 pneumonia. PLoS One 9:e93885. https://doi.org/10.1371/journal.pone. 0093885

24. Wu C, Chen X, Cai Y et al (2020) Risk factors associated with acute respiratory distress syndrome and death in patients with coronavirus disease 2019 pneumonia in Wuhan, China. JAMA Intern Med. https://doi.org/10.1001/jamainternmed.2020.0994

25. Chen R, Liang W, Jiang M, et al (2020) Risk Factors of Fatal Outcome in Hospitalized Subjects With Coronavirus Disease 2019 From a Nationwide Analysis in China Chest 158(1):97-105 https://doi.org/10.1016/j.chest.2020.04.010

26. Lippi G, Favaloro EJ (2020) D-dimer is associated with severity of coronavirus disease 2019: a pooled analysis. Thromb Haemost 120: 876-878. https://doi.org/10.1055/s-0040-1709650

27. Liu F, Li L, Xu M et al (2020) Prognostic value of interleukin-6, Creactive protein, and procalcitonin in patients with COVID-19. J Clin Virol 127:104370. https://doi.org/10.1016/j.jcv.2020.104370

28. Li K, Fang Y, Li W et al (2020) CT image visual quantitative evaluation and clinical classification of coronavirus disease (COVID-19). Eur Radiol. https://doi.org/10.1007/s00330-02006817-6

29. ACR Recommendations for the use of Chest Radiography and Computed Tomography (CT) for Suspected COVID-19 Infection. https://www.acr.org/Advocacy-and-Economics/ACR-PositionStatements/Recommendations-for-Chest-Radiography-and-CTfor-Suspected-COVID19-Infection. Accessed 28 Mar 2020

30. Orsi MA, Oliva AG, Cellina M (2020) Radiology department preparedness for COVID-19: facing an unexpected outbreak of the disease. Radiology 295:E8-E8. https://doi.org/10.1148/radiol. 2020201214

Publisher's note Springer Nature remains neutral with regard to jurisdictional claims in published maps and institutional affiliations. 